\title{
Executive Function Assessment in Patients With Idiopathic Generalized Epilepsy: Applyingthe Frontal Assessment Battery
}

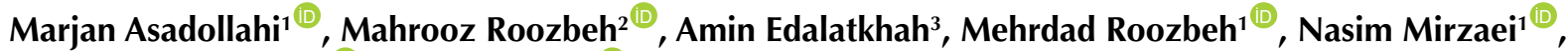 \\ Mohammad Rostami ${ }^{\circledR}$, Leila Simani ${ }^{5,6^{*}}$ \\ ${ }^{1}$ Department of epilepsy, Loghman Hakim Hospital, Shahid Beheshti University of Medical Sciences, Tehran, Iran \\ ${ }^{2}$ Institute for Cognitive and Brain Science, Shahid Beheshti University, Tehran, Iran \\ ${ }^{3}$ Hamava Institute for psychoanalyst psychotherapy, Tehran, Iran \\ ${ }^{4}$ Advanced Medical Technologies and Equipment Institute. Tehran University of Medical Sciences, Tehran, Iran \\ ${ }^{5}$ Brain Mapping Research Center, Shahid Beheshti University of Medical Sciences, Tehran, Iran \\ ${ }^{6}$ Skull Base Research Center, Loghman Hakim Hospital, Shahid Beheshti University of Medical Sciences, Tehran, Iran
}

\begin{abstract}
Background: Executive dysfunction is seen in idiopathic generalized epilepsy (IGE). The Frontal Assessment Battery $(\mathrm{FAB})$ is a short neuropsychological instrument designed in clinical settings to evaluate frontal lobe activity. We aimed to assess the clinical use of FAB in patients with IGE for to detect executive impairment.

Method: In this study, 30 patients with IGE and 30 age- and sex-matched healthy controls were included. The presence and severity of executive dysfunction was investigated with FAB. Cognitive flexibility, decision making, working memory, and general intelligence level were examined using Wisconsin Card Sorting Task (WCST), lowa Gambling Task (IGT), N-back, and Wechsler Adult Intelligence Scale, respectively. In patients with IGE, FAB results were related to their neuropsychological task performance.

Results: The FAB score in patients with IGE was significantly lower compared to healthy participants. In motor programming tasks, patients with IGE performed substantially worse. However, no correlation was found between FAB and neuropsychological task and clinical characteristics.

Conclusion: Executive dysfunction was present in patients with IGE and FAB may be used in these patients as an effective tool for evaluating frontal lobe function.

Keywords: Idiopathic generalized epilepsy; Executive function; Frontal Assessment Battery.
\end{abstract}

\section{*Correspondence to} Leila Simani,

PhD; Skull Base Research Center, Loghman Hakim Hospital, Shahid Beheshti University of Medical Sciences, Kamali St, South Kargar Ave, Tehran, Iran.

Postal code: 1333635445 . Tel: +98-2151025749 Fax: +98-2155416130; Email: I.simani62@gmail.com I.simani90@sbmu.ac.ir

Published online March 27 2021

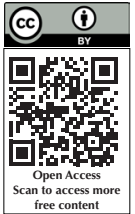

Citation: Asadollahi M, Roozbeh M, Edalatkhah A, Roozbeh M, Mirzaei N, Rostami M, et al. Executive function assessment in patients with idiopathic generalized epilepsy: applyingthe frontal assessment battery. Clin Neurosci J. 2021;8(2):80-84. doi:10.34172/ icnj.2021.17.

\section{Introduction}

Idiopathic generalized epilepsies (IGEs) are a group of epilepsy disorders with presumed genetic etiology. Its characteristic electroencephalography (EEG) pattern consists of normal background activity intermingled with generalized frontal dominant $>3 \mathrm{~Hz}$ spike-wave (SW) or polyspike-wave (PSW) discharges. ${ }^{1}$ GGE accounts for about $15 \%-20 \%$ of all epilepsies. ${ }^{2}$

The potency to manipulate complicated, goal-oriented thoughts and behaviors is an executive function (EF) or cognitive regulation. To achieve this goal, a broad range of cognitive domains are required, including focus, inhibition control, concept formation, planning, working memory, problem solving, and mental flexibility. ${ }^{3} \mathrm{EF}$ is a higher level of cognitive functioning, focusing largely on the function of the frontal lobe. ${ }^{3}$ Previous neuropsychological evaluations and novel neuroimaging techniques in patients with IGE demonstrated evidences of frontal lobe dysfunction. ${ }^{4,5}$ Neuropsychological assessment profile in adult patients with IGE have a wide variety of deficits of frontal lobe function. ${ }^{6,7}$

Frontal Assessment Battery (FAB) was designed to assess frontal lobe impairment in a diversity of patients as a rapid and convenient clinical test. ${ }^{8}$ FAB is divided into six subtests, each of which assesses the EF that is assumed to rely on the frontal cortex (conceptualization, mental flexibility, motor programming, interference, inhibitory control, and environmental autonomy). ${ }^{9}$

FAB has been commonly used in a number of various neurological disorders in recent years, including frontotemporal dementia, ${ }^{10}$ Huntington's disease, ${ }^{11}$ Parkinson's disease, ${ }^{12}$ Alzheimer's disease,${ }^{13}$ temporal lobe epilepsy, ${ }^{14}$ and juvenile myoclonic epilepsy (JME). ${ }^{15} \mathrm{~A}$ brain imaging study demonstrated association between

(C) 2021 The Author(s). This is an open access article distributed under the terms of the Creative Commons Attribution License (http:// creativecommons.org/licenses/by/4.0/), which permits unrestricted use, distribution, and reproduction in any medium, provided the original work is properly cited. 
FAB results and perfusion in the medial and dorsolateral frontal cortex. ${ }^{16}$ Compared to healthy controls, patients with JME showed lower scores in conceptualization, mental flexibility, programming, sensitivity to interference, and total FAB score. ${ }^{15}$ However, association between FAB and formal EF measurements have not been investigated specially in subtypes of patients with IGE. We aimed to apply FAB with other EF tests in patients with IGE and compare it with healthy individuals.

\section{Materials and Methods \\ Participants}

This analytical cross-sectional study was performed on 30 patients diagnosed with IGE, and 30 healthy individuals. According to the International League Against Epilepsy (ILAE) definitions, IGE syndromes are classified into four groups, based on clinical history and seizure types, as follows: childhood absences epilepsy (CAE), juvenile absences epilepsy (JAE), JME, and epilepsy with generalized tonic-clonic seizures alone (GTCS-alone) ${ }^{17}$ Patients in the IGE group had at least one recorded EEG with typical normal background rhythm intermingled with frontally predominant generalized $>3 \mathrm{~Hz}$ SW or/and PSW discharges. Age, sex, duration of epilepsy, types of seizures, frequency of attacks, and risk factors for epilepsy and the medication history were determined. Exclusion criteria were: $(a)$ patients under the age of 18 or over 50 years of age, $(b)$ those with other medical, neurological (Parkinson's disease, dementia, stroke, etc), and psychological conditions (schizophrenia, bipolar mood disorder, etc), or history of recent head trauma or those with intellectual disability. Healthy volunteers without a history of seizures were included in the control group.

\section{Neuropsychological Tests}

According to previous research, we selected an extensive battery of neuropsychiatric tests (table 2) that were stated to be useful in patients with IGE: Wisconsin Card Sorting Task (WCST, Cognitive flexibility), Iowa Gambling Task (IGT, decision making), and N-back (working memory). Based on a wide sample of healthy participants, all the chosen assessments had accurate scoring parameters, short administration time, and were standardized.

The FAB has previously been validated in Persian. ${ }^{18}$ Each test item is counted from 0 to 3 ; the overall score ranges from 0 to 18 and shows executive impairment and its severity. In this study, the six subsets of this method were evaluated as follows: (a) Similarities (conceptualization), (b) Lexical fluency (mental flexibility), (c) Motor series "Luria" test (programming), (d) Conflicting instructions (sensitivity to interference), (e) Go-No-Go (inhibitory control), ( $f$ ) Pretension behavior (environmental autonomy)

\section{Statistical Analysis}

Statistical analyses were performed using SSPS software, version 22.0 (SPSS, Inc., Chicago, IL, USA). To evaluate normal data distributions, Kolmogorov-Smirnov test was used. Chi-square and independent $t$ test were applied to analyze categorical and numerical data, respectively. Group differences (in IGE and healthy control (HC) s) for each neuropsychological tasks were compared using independent $t$ test. To determine the strength and direction of the linear correlation between variables, Pearson correlation coefficient was calculated. Spearman rank coefficient was applied when non-normality data was present. $P<0.05$ was estimated as significant.

\section{Results}

In this study, 30 patients with IGE and 30 controls were included. The clinical and demographic characteristics of the patients are shown in Table 1. In terms of age, sex, and level of education there were no significant differences between the two groups.

In contrast to healthy controls, the overall $\mathrm{FAB}$ rates were noticeably lower in patients with IGE $(P<0.04)$. As shown in Table 2, significantly lower scores in the motor programming subtest were obtained from patients with IGE $(P<0.01)$.

Student's $t$ test showed significant differences in the WCST test between patients with IGE and healthy controls. Patients with IGE had more preservative errors in WCST $(P=0.04)$. The two studied groups did not differ significantly in IQ, N-Back, and IGT $(P>0.05)$. The mean scores for every test are shown in Table 2. No correlation was observed between FAB and the clinical characteristics

Table 1. Clinical Characteristics of Participants in the Study

\begin{tabular}{lccc}
\hline Variables & IGE (30) & HC (30) & P Value \\
\hline Age & $27.63 \pm 6$ & $31.10 \pm 1$ & 0.06 \\
Sex (male/female) & $10 / 20$ & $14 / 16$ & 0.292 \\
Level of education & & & \\
Primary & $10(33.3)$ & $7(23.3)$ & 0.684 \\
High School & $10(33.3)$ & $12(40)$ & \\
Academic & $10(33.3)$ & $11(36.7)$ & \\
Disease Duration (y) & $11.13 \pm 9.39$ & & \\
Frequency of Seizure (per month) & $2.86 \pm 1.25$ & & \\
Diagnosis of Epilepsy & & \\
JAE & $7(23.3)$ & \\
JME & $11(36.7)$ & \\
GTCS alone & $12(40.0)$ & \\
AED therapy & & \\
Monotherapy & & \\
Polytherapy & $82(73.3)$ & \\
\hline Abbreviation: IGE, idiopathic generalized epilepsy; AFD, Anti-epileptic drug; \\
JAE, juvenile absences epilepsy; JME, juvenile myoclonic epilepsy; HC, \\
healthy control
\end{tabular}


Table 2. The Mean \pm SD of Neuropsychological Task

\begin{tabular}{|c|c|c|c|}
\hline Variables & IGE & $\mathrm{HC}$ & $P$ Value \\
\hline Intelligent quotient & $89.13 \pm 11.67$ & $88.26 \pm 8.1$ & 0.112 \\
\hline \multicolumn{4}{|l|}{ WCST } \\
\hline Categories achieved & $3.33 \pm 2.1$ & $3.89 \pm 1.79$ & 0.279 \\
\hline Preservative errors & $6.86 \pm 4.76$ & $4.62 \pm 3.66$ & $0.048^{*}$ \\
\hline \multicolumn{4}{|l|}{ N-Back } \\
\hline Reaction times & $635.76 \pm 101.87$ & $611.62 \pm 138.56$ & 0.448 \\
\hline Correct response & $89.03 \pm 26.06$ & $94.51 \pm 29.90$ & 0.455 \\
\hline \multicolumn{4}{|l|}{ IGT } \\
\hline Gain & $9578.33 \pm 532.03$ & $9400.0 \pm 528.47$ & 0.254 \\
\hline Loss & $7903.33 \pm 1297.07$ & $7782.75 \pm 956.83$ & 0.687 \\
\hline Total FAB score & $15.86 \pm 1.62$ & $16.73 \pm 0.9$ & $0.04^{*}$ \\
\hline Conceptualization & $2.86 \pm 0.3$ & $2.90 \pm 0.3$ & 0.694 \\
\hline Mental flexibility & $2.23 \pm 0.6$ & $2.43 \pm 0.6$ & 0.24 \\
\hline Motor programming & $2.66 \pm 0.4$ & $2.93 \pm 0.2$ & $0.01^{*}$ \\
\hline Sensitivity interference & $2.50 \pm 0.5$ & $2.66 \pm 0.4$ & 0.197 \\
\hline Inhibitory control & $2.56 \pm 0.5$ & $2.80 \pm 0.4$ & 0.05 \\
\hline Environmental autonomy & $2.86 \pm 0.3$ & $2.90 \pm 0.3$ & 0.694 \\
\hline
\end{tabular}

Abbreviations: IGE, idiopathic generalized epilepsy; WCST, Wisconsin Card Sorting Task; IGT, lowa Gambling Task; FAB, Frontal Assessment Battery; HC, healthy control

* Significant $<0.05$

of patients with IGE. Moreover, the neuropsychological tasks were not affected by the subtype of IGE (including JAE, JME and GTCS alone) or patients' anti-seizure medications.

\section{Discussion}

The findings of this study are compatible with previous reports indicating impaired frontal lobe function in patients with IGE. ${ }^{15,19}$ The major findings of this study are as follows: (1) compared with healthy controls, patients with IGE showed lower total FAB score and lower score in motor programming; (2) disease duration and frequency of seizure had no effect on FAB scores in patients with IGE and EF tasks; (3) the number of AEDs (polytherapy versus monotherapy) and subtypes of IGE had no significant effect on FAB scores and EF tasks; and (4) Compared with healthy participants, the number of preservative errors in WCST in patients with IGE were lower. It suggests that this battery has good validity as a discriminant.

Most previous studies evaluated EF by the Stroop test, the verbal fluency test, the digit span tests, the WCST, and the N-back, which are the gold standard for IGE executive skills assessment. ${ }^{20-23}$ However, more recent studies showed that the mentioned tests are not specific for frontal lobe functions. ${ }^{24}$ While frontal and prefrontal cortical areas play important roles in the success of the task, other brain areas are also largely involved, including the inferior parietal lobe, temporoparietal junction, and visual processing areas. ${ }^{24-26}$

The most commonly recorded impaired IGE skills are deficient working memory, impaired verbal fluency tasks, and language deficits, which are particularly, though not mainly, important for optimal frontal lobe activity. ${ }^{19,20,27}$ However, previous studies have also identified problems in inhibitory control, and other main domains of EF. ${ }^{4,28}$

Typically, IGE is correlated with general intelligence, although several patients show different cognitive deficiencies in the frontal lobe. ${ }^{29,30}$ A meta-analysis found that except for visual-spatial skills, patients with IGE displayed substantially lower scores in all cognitive domains. ${ }^{19}$ The higher seizure frequency was associated with lower test scores. ${ }^{31}$

Interictal epileptiform discharges (IEDs) have been proposed to lead to frontal lobe dysfunction in patients with IGE. In a study by Jiang et al, as compared to patients without IEDs, IGE patients with IEDs conducted poorly on EF tests. Moreover, patients with IGE without IEDs performed worse than healthy controls. ${ }^{32}$

There may be many reasons for these variations in outcomes. The complexity of the EFs could be one reason and the fact that not all the domains be necessary involved. In addition, because of the genotype and phenotype heterogeneity of the patients, the performance of all of them may not be uniform. Even some patients showed little or no significant deficits in the EFs. ${ }^{29,33}$

Many studies have found that EFs are involved in patients with $\mathrm{JME}^{29,34,35}$ and only a few studied patients with other forms of IGE syndrome. ${ }^{4,36,37}$ As a result, it seems important to provide more data on the EFs in IGE syndromes other than JME. In our study, the EFs have 
been assessed in the subgroups of patients with IGE, and no variations between the groups have been observed in any of the tasks.

Although the dosage and number of AEDs raised the risk of cognitive dysfunction, ${ }^{38}$ polytherapy in this study was not related to a more impaired EF. The main side effects of AEDs were memory deficiency and executive dysfunction. Older anticonvulsants tend to compromise cognition more than newer agents. ${ }^{39}$

Neuropsychological and functional imaging tests have demonstrated cognitive dysfunction in the frontal lobe. Microstructural abnormalities have been defined on the supplementary motor area as a key hub in a thalamofrontal cortical network. ${ }^{40,41}$ At a higher cognitive load, frontal lobe dysfunction tends to become more noticeable, which may account for the variability between the "normal "output results in patients with IGE on tasks that are not sufficiently difficult.

\section{Conclusion}

Although, IGE harbors a benign course, the probability of executive dysfunction was reported in these patients. In our study, impaired motor programing and preservative error were found in patients with IGE compared to the controls. The executive dysfunction in patients with IGE was not correlated with the epilepsy duration, types and dosage of anti-seizure medications, or IGE subtypes. Epilepsy is a disorder that affects widespread brain network rather than a discrete brain region. We proposed that $\mathrm{FAB}$ is a clinically applicable instrument for monitoring EF in patients with IGE. In the period of the disease, early neuropsychological evaluation may provide appropriate intervention where necessary.

\section{Conflict of Interest}

None of the authors has any conflict of interest to disclose.

\section{Acknowledgments}

The authors would like to thank the Clinical Research Development Unit (CRDU) of Loghman Hakim hospital, Shahid Beheshti University of Medical Sciences, Tehran, Iran for their support, cooperation, and assistance throughout the study.

\section{Authors' Contribution}

Study concept and design: LS, and MA. Acquiring data: MR, Amin Edalatkhah, MR, NM. Analysis and interpretation of data: LS and MR. Drafting of the manuscript: LS and MR. Critical revision of the manuscript for important intellectual content: LS and MA. Acqiring data: $\mathrm{AE}$

\section{Funding/Support}

This research did not receive any specific grant from funding agencies in the public, commercial or not-for-profit sectors.

\section{Ethics Statement}

The study has been approved by the Ethics Committee of Shahid Beheshti University of Medical Sciences, Tehran, Iran (Number: IR.SBMU.MSP.REC.1398. 41).

\section{Ethical Statement}

The study has been approved by the Ethics Committee of Shahid Beheshti University of Medical Sciences, Tehran, Iran (Number: IR.SBMU.MSP.REC.1398. 41).

\section{References}

1. Berg AT, Berkovic SF, Brodie MJ, Buchhalter J, Cross JH, van Emde Boas W, et al. Revised terminology and concepts for organization of seizures and epilepsies: report of the ILAE Commission on Classification and Terminology, 20052009. Epilepsia. 2010;51(4):676-85. doi: 10.1111/j.15281167.2010.02522.x.

2. Jallon P, Latour P. Epidemiology of idiopathic generalized epilepsies. Epilepsia. 2005;46 Suppl 9:10-4. doi: 10.1111/j.1528-1167.2005.00309.x.

3. Diamond A. Executive functions. Annu Rev Psychol. 2013;64:135-68. doi: 10.1146/annurevpsych-113011-143750.

4. Simani L, Roozbeh M, Rostami M, Pakdaman H, Ramezani $\mathrm{M}$, Asadollahi M. Attention and inhibitory control deficits in patients with genetic generalized epilepsy and psychogenic nonepileptic seizure. Epilepsy Behav. 2020;102:106672. doi: 10.1016/j.yebeh.2019.106672.

5. Simani L, Raminfard S, Asadollahi M, Roozbeh M, Ryan F, Rostami M. Neurochemicals of limbic system and thalamofrontal cortical network: are they different between patients with idiopathic generalized epilepsy and psychogenic nonepileptic seizure? Epilepsy Behav. 2020;112:107480. doi: 10.1016/j.yebeh.2020.107480.

6. Elger CE, Helmstaedter C, Kurthen M. Chronic epilepsy and cognition. Lancet Neurol. 2004;3(11):663-72. doi: 10.1016/ s1474-4422(04)00906-8.

7. Hommet C, Sauerwein HC, De Toffol B, Lassonde M. Idiopathic epileptic syndromes and cognition. Neurosci Biobehav Rev. 2006;30(1):85-96. doi: 10.1016/j.neubiorev.2005.06.004.

8. Dubois B, Slachevsky A, Litvan I, Pillon B. The FAB: a Frontal Assessment Battery at bedside. Neurology. 2000;55(11):16216. doi: 10.1212/wnl.55.11.1621.

9. Moorhouse P, Gorman M, Rockwood K. Comparison of EXIT-25 and the Frontal Assessment Battery for evaluation of executive dysfunction in patients attending a memory clinic. Dement Geriatr Cogn Disord. 2009;27(5):424-8. doi: $10.1159 / 000212755$.

10. Slachevsky A, Villalpando JM, Sarazin M, Hahn-Barma V, Pillon B, Dubois B. Frontal assessment battery and differential diagnosis of frontotemporal dementia and Alzheimer disease. Arch Neurol. 2004;61(7):1104-7. doi: 10.1001/ archneur.61.7.1104.

11. Rodrigues GR, Souza CP, Cetlin RS, de Oliveira DS, PenaPereira M, Ujikawa LT, et al. Use of the frontal assessment battery in evaluating executive dysfunction in patients with Huntington's disease. J Neurol. 2009;256(11):1809-15. doi: 10.1007/s00415-009-5197-0.

12. Lima CF, Meireles LP, Fonseca R, Castro SL, Garrett C. The Frontal Assessment Battery (FAB) in Parkinson's disease and correlations with formal measures of executive functioning. J Neurol. 2008;255(11):1756-61. doi: 10.1007/s00415-0080024-6.

13. Nagata $T$, Shinagawa $S$, Ochiai $Y$, Aoki R, Kasahara H, Nukariya $\mathrm{K}$, et al. Association between executive dysfunction and hippocampal volume in Alzheimer's disease. Int Psychogeriatr. 2011;23(5):764-71. doi: 10.1017/s1041610210002164. 
14. Agah E, Asgari-Rad N, Ahmadi M, Tafakhori A, Aghamollaii V. Evaluating executive function in patients with temporal lobe epilepsy using the Frontal Assessment Battery. Epilepsy Res. 2017;133:22-7. doi: 10.1016/j.eplepsyres.2017.03.011.

15. Sanjari Moghaddam H, Doost Hoseini M, Khaleghi MR, Tafakhori A, Dolatshahi M, Pourmirbabaei S, et al. Evaluating executive functions in patients with juvenile myoclonic epilepsy using Frontal Assessment Battery. Behav Neurol. 2020;2020:8710373. doi: 10.1155/2020/8710373.

16. Guedj E, Allali G, Goetz C, Le Ber I, Volteau M, Lacomblez $\mathrm{L}$, et al. Frontal Assessment Battery is a marker of dorsolateral and medial frontal functions: a SPECT study in frontotemporal dementia. J Neurol Sci. 2008;273(1-2):84-7. doi: 10.1016/j. jns.2008.06.035.

17. Scheffer IE, Berkovic S, Capovilla G, Connolly MB, French J, Guilhoto L, et al. ILAE classification of the epilepsies: position paper of the ILAE Commission for Classification and Terminology. Epilepsia. 2017;58(4):512-21. doi: 10.1111/ epi.13709.

18. Asaadi S, Ashrafi F, Omidbeigi M, Nasiri Z, Pakdaman H, Amini-Harandi A. Persian version of frontal assessment battery: correlations with formal measures of executive functioning and providing normative data for Persian population. Iran J Neurol. 2016;15(1):16-22.

19. Loughman A, Bowden SC, D'Souza W. Cognitive functioning in idiopathic generalised epilepsies: a systematic review and meta-analysis. Neurosci Biobehav Rev. 2014;43:20-34. doi: 10.1016/j.neubiorev.2014.02.012.

20. Chowdhury FA, Elwes RD, Koutroumanidis M, Morris RG, Nashef L, Richardson MP. Impaired cognitive function in idiopathic generalized epilepsy and unaffected family members: an epilepsy endophenotype. Epilepsia. 2014;55(6):835-40. doi: 10.1111/epi.12604.

21. Rai VK, Shukla G, Afsar M, Poornima S, Pandey RM, Rai N, et al. Memory, executive function and language function are similarly impaired in both temporal and extra temporal refractory epilepsy-a prospective study. Epilepsy Res. 2015;109:72-80. doi: 10.1016/j.eplepsyres.2014.09.031.

22. de Lima AB, Moreira F, da Mota Gomes M, Maia-Filho $\mathrm{H}$. Clinical and neuropsychological assessment of executive function in a sample of children and adolescents with idiopathic epilepsy. Arq Neuropsiquiatr. 2014;72(12):954-9. doi: 10.1590/0004-282x20140191.

23. Riccio CA, Pliego JA, Cohen MJ, Park Y. Executive function performance for children with epilepsy localized to the frontal or temporal lobes. Appl Neuropsychol Child. 2015;4(4):27784. doi: 10.1080/21622965.2014.923774.

24. Nyhus E, Barceló F. The Wisconsin Card Sorting Test and the cognitive assessment of prefrontal executive functions: a critical update. Brain Cogn. 2009;71(3):437-51. doi: 10.1016/j.bandc.2009.03.005.

25. Lie $\mathrm{CH}$, Specht $\mathrm{K}$, Marshall JC, Fink GR. Using fMRI to decompose the neural processes underlying the Wisconsin Card Sorting Test. Neuroimage. 2006;30(3):1038-49. doi: 10.1016/j.neuroimage.2005.10.031.

26. Seo J, Chang Y, Jang KE, Park JW, Kim YT, Park SJ, et al. Altered executive function in the welders: a functional magnetic resonance imaging study. Neurotoxicol Teratol. 2016;56:2634. doi: 10.1016/j.ntt.2016.05.003.

27. Birn RM, Kenworthy L, Case L, Caravella R, Jones TB, Bandettini PA, et al. Neural systems supporting lexical search guided by letter and semantic category cues: a self-paced overt response
fMRI study of verbal fluency. Neuroimage. 2010;49(1):1099107. doi: 10.1016/j.neuroimage.2009.07.036.

28. Wandschneider B, Thompson PJ, Vollmar C, Koepp MJ. Frontal lobe function and structure in juvenile myoclonic epilepsy: a comprehensive review of neuropsychological and imaging data. Epilepsia. 2012;53(12):2091-8. doi: 10.1111/ epi.12003.

29. Devinsky O, Gershengorn J, Brown E, Perrine K, Vazquez B, Luciano D. Frontal functions in juvenile myoclonic epilepsy. Neuropsychiatry Neuropsychol Behav Neurol. 1997; 10(4):243-6.

30. Kim JH, Suh SI, Park SY, Seo WK, Koh I, Koh SB, et al. Microstructural white matter abnormality and frontal cognitive dysfunctions in juvenile myoclonic epilepsy. Epilepsia. 2012;53(8):1371-8. doi: 10.1111/j.1528-1167.2012.03544.x.

31. Gupta S, Kwan P, Faught E, Tsong W, Forsythe A, Ryvlin P. Understanding the burden of idiopathic generalized epilepsy in the United States, Europe, and Brazil: an analysis from the National Health and Wellness Survey. Epilepsy Behav. 2016;55:146-56. doi: 10.1016/j.yebeh.2015.12.018.

32. Jiang Y, Wu J, Wang Y, Zhou N, Wang K. Impaired executive function by interictal epileptiform discharges in patients with idiopathic generalized epilepsy. Epilepsy J. 2017;3(1):118. doi: 10.4172/2472-0895.1000118.

33. Mefford HC, Muhle H, Ostertag P, von Spiczak S, Buysse $\mathrm{K}$, Baker $\mathrm{C}$, et al. Genome-wide copy number variation in epilepsy: novel susceptibility loci in idiopathic generalized and focal epilepsies. PLoS Genet. 2010;6(5):e1000962. doi: 10.1371/journal.pgen.1000962.

34. Meador KJ. Brain function and anatomy in juvenile myoclonic epilepsy. Epilepsy Curr. 2010;10(1):13-4. doi: 10.1111/j.15357511.2009.01340.x.

35. Pulsipher DT, Seidenberg M, Guidotti L, Tuchscherer VN, Morton J, Sheth RD, et al. Thalamofrontal circuitry and executive dysfunction in recent-onset juvenile myoclonic epilepsy. Epilepsia. 2009;50(5):1210-9. doi: 10.1111/j.15281167.2008.01952.x.

36. Høie B, Sommerfelt K, Waaler PE, Alsaker FD, Skeidsvoll $\mathrm{H}$, Mykletun A. Psychosocial problems and seizure-related factors in children with epilepsy. Dev Med Child Neurol. 2006;48(3):213-9. doi: 10.1017/s0012162206000454.

37. Taylor J, Kolamunnage-Dona R, Marson AG, Smith PE, Aldenkamp AP, Baker GA. Patients with epilepsy: cognitively compromised before the start of antiepileptic drug treatment? Epilepsia. 2010;51(1):48-56. doi: 10.1111/j.15281167.2009.02195.x.

38. Witt JA, Elger CE, Helmstaedter C. Adverse cognitive effects of antiepileptic pharmacotherapy: each additional drug matters. Eur Neuropsychopharmacol. 2015;25(11):1954-9. doi: 10.1016/j.euroneuro.2015.07.027.

39. Eddy CM, Rickards HE, Cavanna AE. The cognitive impact of antiepileptic drugs. Ther Adv Neurol Disord. 2011;4(6):385407. doi: 10.1177/1756285611417920.

40. Woermann FG, Free SL, Koepp MJ, Sisodiya SM, Duncan JS. Abnormal cerebral structure in juvenile myoclonic epilepsy demonstrated with voxel-based analysis of MRI. Brain. 1999;122(Pt 11):2101-8. doi: 10.1093/brain/122.11.2101.

41. Vollmar C, O'Muircheartaigh J, Symms MR, Barker GJ, Thompson P, Kumari $\mathrm{V}$, et al. Altered microstructural connectivity in juvenile myoclonic epilepsy: the missing link. Neurology. 2012;78(20):1555-9. doi: 10.1212/ WNL.0b013e3182563b44. 